\title{
Percrestal Sinuslift: From Illusion to Reality
}
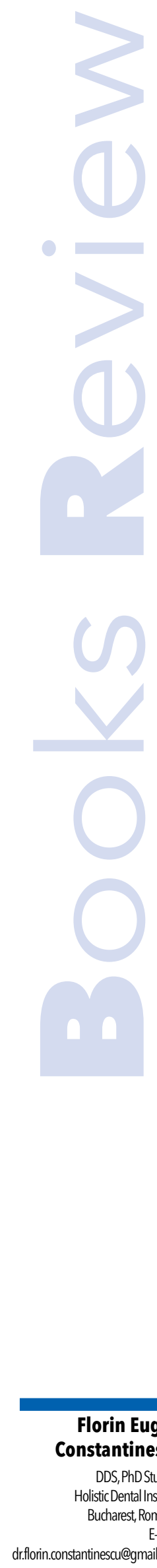

Florin Eugen Constantinescu

DDS, PhDStudent

Holistic Dental Institute

Bucharest, Romania

E-mail:

dr.florin.constantinescu@gmail.com

\section{George Watzek}

Quintessence Publishing Company, Inc

Language: English

248 pages, 535 illustrations, DVD included

ISBN: 978-1-85097-222-8

Publication Date: December, 2011

Price: $128.00 €$

Minimally invasive surgical techniques are used more and more in medicine. Based on the popularity gained by their introduction in medicine, the 11 contributors of Bernhard Gottlieb School of Dentistry at the Medical University of Vienna under the direction of Professor George Watzek give us a guide for transcrestal sinus floor elevation in oral implantology of the posterior maxillary. The book is divided into 12 chapters.

In the first chapter, "Maxillary sinus anatomy and physiology", the authors present the morphologic variability, the innervation and blood supply, the sinus ventilation and the mucociliary activity.

The second chapter, "Biological Aspects of sinus augmentation", describes the histology of bone regeneration in the augmented sinus, regeneration and repair, mechanically stable conditions: a key factor of bone regeneration, angiogenesis: a key factor of bone regeneration, configuration changes of the augmented sinus, form follows function, augmented sinus and principle of guided bone regeneration. Bone morphogenetic proteins: osteoinductive growth factors, platelet-derived growth factor-BB and PRP: non - osteoinductive growth factors, cell therapy in sinus augmentation, compromised bone regeneration: impact on graft consolidation and current knowledge and future perspectives are presented.

In Chapter 3, "Generally accepted procedures" and in Chapter 4, "Status quo analysis", techniques of bone instrumentation, techniques for elevating the sinus membrane and methods of assessing the membrane integrity are described.

Chapter 5, "Biomechanics of transcrestal sinus membrane elevation", describes transcrestal membrane elevation techniques, the biomechanical properties of the maxillary sinus membrane, the transmission of elevation forces and the impact of internal sinus membrane elevation anatomy and patterns with multiple osteotomies.

Chapter 6, "Radiologic assessment", describes the general and specific preoperative assessment, the intraoperative imaging and the postoperative imaging.

Chapter 7, "Preoperative measures for assuring success", contains local preoperative diagnostic work-up, local treatment modalities and the general preoperative work-up.

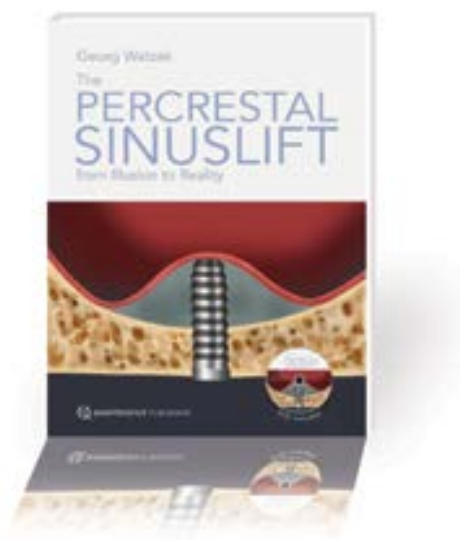

Chapter8, "Transcrestalosteotomy: technological considerations and options for bone perforation", presents the osteotome technique, the drill osteotomy technique, the ultrasonic technique and the laser osteotomy technique.

Chapter 9, "Insights into sinus augmentation: preclinical and clinical research", outlines general aspects of sinus augmentation and terminology, preclinical and clinical models for investigating graft consolidation, graft consolidation gradient (GCG), injectable grafts, combination of grafts and growth factors and combination of grafts and cells.

In Chapter 10, "Clinicalexperiencesusing innovative equipment", the preoperative planning, trephination of the bony sinus floor, liquid-pressure-mediated membrane elevation, intraoperative evidence of iatrogenic membrane perforation, implant placement and postoperative procedures, clinical results and clinical considerations are described.

In Chapter 11 the "Compromised results and complications" are analyzed: sinus membrane injury and its consequences, dealing with a perforated sinus membrane, problems of membrane elevation, problems of grafting, implant placement and potential problems of maxillary sinusitis.

In the last chapter, "Summary and Outlook", Professor Watzek makes a synthesis on transcrestal sinus floor elevation in terms of a flapless minimally invasive procedure, concluding that for a good success of the method we must use three-dimensional X-ray imaging and a minimum volume of elevating liquid.

This book is an excellent guide for both the experienced oral implantologist and the beginner, who want more information on sinus augmentation. The text contains 535 color illustrations that make it easy to follow. It is accompanied by a DVD - ROM including images of percrestal sinuslift surgery using the pressure gel technique. The book of Professor Watzek is a documentary source indispensable for any oral implantologist that wants to successfully practice the technique of transcrestal sinus floor elevation. 